\title{
la Cualidad Humana y la Cualidad Humana Profunda EN LAS SOCIEDADES AFECTADAS POR LA DinÁMICA ACELERADA DE LAS TECNOCIENCIAS
}

\author{
THE Human Quality and THE DeEP Human Quality IN SOCIETIES \\ AFFECTED BY THE ACCELERATED DYNAMICS OF THECNOSCIENCES
}

M. CORBí ${ }^{1}$

\begin{abstract}
RESUMEN
Hace pocas décadas que una dinámica de crecimiento acelerado de las tecnociencias en retroalimentación mutua, capaz de generar la aparición constante y acelerada de nuevos productos y servicios, está invadiendo todas las sociedades humanas y afectando a las maneras de vivir, porque altera los proyectos axiológicos colectivos de tal forma que debilita y corroe todas las instituciones, incluidas las religiones. Esta dinámica imparable nos fuerza a advertir que tenemos un doble acceso a la realidad, uno relativo a nuestras necesidades y otro no relativo, absoluto. Nos fuerza además a cambiar nuestra concepción de la epistemología, de la antropología y de la espiritualidad. Lo que nuestros antepasados llamaron "espiritualidad", nosotros hemos de llamarlo, de acuerdo a la nueva antropología, "cualidad humana profunda". Esta espiritualidad está pasando, por el empuje de las sociedades de conocimiento, de la práctica que transita por la sumisión, a la práctica que transita por la indagación libre. Siempre apoyada y orientada por la sabiduría de nuestros antepasados. Una espiritualidad de dos pisos, el humano y el divino, se transforma en una espiritualidad en la que la realidad que vivimos, siendo una, se presenta con doble dimensión: la relativa y la absoluta.
\end{abstract}

Palabras Clave: Sociedad de conocimiento, espiritualidad, cualidad humana, cualidad humana profunda, epistemología axiológica.

\begin{abstract}
Over the past few decades, the rapidly growing mutual feedback dynamic of the technosciences, capable of generating constant and rapid emergence of new products and services, has invaded all human societies and affected our ways of life because it has altered collective axiological projects in such a way as to weaken and corrode all institutions, including religious ones. This unstoppable technoscientific dynamism forces us to acknowledge that we have a dual access to the real: one related to our needs and another not relative, but absolute. It also forces us to change our conceptions of epistemology, anthropology and spirituality.

That which our ancestors named "spirituality" we must call it, in accordance with the new anthropology, "a deep human quality." Induced by knowledge societies, this spirituality is transforming from being a practice channelled through submission to one channelled through open inquiry, always supported and guided by the wisdom of our forefathers. Thus, a spirituality communicating two levels, the human and the divine, becomes a spirituality in which the reality we live, being unique, presents itself through two dimensions: the relative and the absolute.
\end{abstract}

KEYWORDS: Knowledge society, spirituality, human quality, deep human quality, axiological epistemology.

\footnotetext{
${ }^{1}$ Director del Centro de Estudio de las Tradiciones de Sabiduría (CETR) de Barcelona. Licenciado en Teología y Doctor en Filosofía, ha sido Profesor de ESADE, en la Fundación Vidal y Barraquer y en el Instituto de Teología Fundamental de Barcelona. Epistemólogo investigador de las formaciones axiológicas. País de origem: Espanha.E-mail mcorbi@cetr.net
} 


\section{INTRODUCCIÓN}

Desde hace pocas décadas se ha ido introduciendo una nueva dinámica en los modo de sobrevivencia humana: se pretende sacar provecho para sobrevivir y mejorar mediante la creación de nuevas ciencias, nuevas tecnologías y, a través de ellas, crear, para el servicio de todas las dimensiones de la vida humana, nuevos productos y nuevos servicios. Y esto de una manera continuada y progresivamente acelerada.

\section{LA NUEVA DINÁMICA DE LAS TECNOCIENCIAS Y SUS CONSECUENCIAS.}

Esta dinámica cuando está plenamente desarrollada la llamamos "sociedades de conocimiento". Las sociedades de conocimiento no se han extendido mucho todavía, pero la dinámica acelerada del desarrollo de las tecnociencias y sus consecuencias ya ha invadido prácticamente a todos los pueblos.

Esta continua innovación está desarticulando, en la medida en que se introduce, todos los modos de vida de los individuos y de los colectivos. Especialmente está creado un grave problema en los sistemas de motivación y cohesión colectiva. Esta es razón de la gran crisis de valores.

La crisis está clara en el ámbito religioso, en los sistemas de valores colectivos, en el orden económico, en las organizaciones políticas, en la familia, en las relaciones sexuales, en las relaciones internacionales, en la ecología, en las empresas, etc.

Este modo de sobrevivencia, que se va introduciendo aceleradamente, genera una nueva dinámica que se va imponiendo como un destino inevitable para toda la humanidad. Esta es la dinámica:

El crecimiento continuo y acelerado de las ciencias conduce a un crecimiento continuo y acelerado de las tecnologías. Se produce un ciclo de retroalimentación mutua entre ciencias y tecnologías que lleva a un crecimiento progresivamente más acelerado. Del crecimiento de las tecnociencias se sigue inevitablemente la creación de nuevos productos y nuevos servicios. Las consecuencias de estos hechos que se siguen de las tecnociencias, alteran necesariamente las formas de trabajar y de organizarse.

Todo ello modifica profundamente los modos de vida y las formas de cohesión y motivación social, es decir, altera los sistemas de valores colectivos y, con ellos, las religiones.

En resumen, la dinámica que generan los desarrollos cada vez más acelerados y de mayor calado de las tecnociencias en interacción mutua, alteran profundamente las maneras de vivir $y$, consiguientemente, las maneras de pensar, de sentir y de actuar y organizarse. 
¿Por qué el desarrollo cada día más acelerado de las ciencias y las técnicas y las consecuencias que acarrean desarticulan la vida de los individuos y los colectivos?

Porque estamos transitando de vivir excluyendo los cambios de importancia, a vivir de la creación del cambio en todos los niveles de la vida. Estamos transitando de fundamentarse en creencias religiosas o laicas que fijaban los modos de vivir mediante la sumisión, a fundamentarnos en la indagación y la creación libres.

Esta nueva actitud indagadora y libre se va introduciendo continua y rápidamente en todas partes, también en los ámbitos religiosos, axiológicos y organizativos.

LAS CONSECUENCIAS DE LA DINÁMICA DE CRECIMIENTO DE LAS TECNOCIENCIAS SE EXTENDERÁN A TODOS LOS PUEBLOS.

El proceso de esa dinámica es como un destino inevitable, no solo para algunos pueblos, sino para toda la humanidad. Se introducen las tecnologías, que arrastran a los modos de vivir y en la misma proporción van minando y corroyendo las formas tradiciones de vivir, pensar y organizarse.

Cuando esto ocurre, ya se ha iniciado un proceso que no tendrá marcha atrás. ¿Hay algún país en la tierra que no haya entrado en esta cadena de transformaciones?

¿Es, pues, un destino inevitable para todos los pueblos? Sí. Cuando aparece un sistema de sobrevivencia en el medio, que resulta más eficaz y beneficioso para los colectivos humanos, como fue la aparición de la agricultura o la industria, todos los pueblos se ven forzados a adoptarlos, si las circunstancias se lo permiten. Podría decirse que ningún colectivo es libre de adoptar o no la nueva forma de vida.

Hay, por lo menos, un par de razones para que se produzca inevitablemente este fenómeno:

Los humanos somos depredadores necesitados; todo lo que asegure mejor nuestra sobrevivencia como grupos, lo adoptamos, si las circunstancias nos lo hacen posible.

Si un pueblo adopta el nuevo modo de vida, fuerza a todos sus vecinos a seguir el mismo camino. Si no lo hacen, el pueblo que lo adopta cobra gran ventaja sobre sus vecinos y, tarde o temprano, se apodera de sus tierras, de sus riquezas, de sus cotos de caza, etc. y los convierte en subordinados a su servicio.

Así ha ocurrido varias veces en la historia: en el paso de cazadores/recolectores a agricultores; de agricultores a industriales; y ahora de 
industriales a sociedades de conocimiento. Las nuevas formas de vida, en los aspectos que son más eficaces para sobrevivir, se adoptan necesariamente. Esto es lo que nos dice la lógica y lo que nos confirma la historia de nuestra especie.

Según esto, cuando aparece la posibilidad de vivir y de progresar creando de forma continuada ciencias, tecnologías, nuevos productos y nuevos servicios, esa posibilidad se convierte, con mayor razón que en el pasado, en un destino inevitable para todos los pueblos.

Es razonable pensar que ocurrirá como en el pasado y que, también como en el pasado, los que no adopten esas nuevas formas de vida o sean lentos y reticentes a hacerlo, sean castigados con la dependencia y la sumisión.

Llevamos varias décadas en las que la dinámica que hemos mentado está operando desarticulando los patrones axiológicos de las sociedades, en todos sus niveles. La globalización ha borrado todas las fronteras, de forma que la corrosión se puede extender fácilmente por todas las civilizaciones y todos los tipos de culturas.

\section{MAL USO DE LAS SOCIEDADES DE CONOCIMIENTO Y SUS CONSECUENCIAS}

A las sociedades que están invadidas por esa dinámica, que comporta una nueva forma de sobrevivir en esta tierra, gracias a la creación continua de conocimientos, les llamaremos "sociedades de conocimiento".

La dinámica acelerada de las tecnociencias y sus consecuencias, en un contexto en el que la globalización ha roto todas las fronteras, está siendo utilizada, desde los grandes centros financieros y manufactureros para la explotación, cada día que pasa más eficiente, del medio físico y de los grupos humanos que todavía no han entrado suficientemente en las sociedades de conocimiento.

Esta explotación intensiva gracias al conocimiento, está dañando al medio, en algunos aspectos de manera irreversible, ha provocado ya la mayor extinción de especies de la historia de la vida, está haciendo el planeta inhabitable y está creando grandes diferencias en la disposición de recursos entre unos pueblos y otros.

Así no podemos seguir, porque se está dañando gravemente a la mayoría de los pueblos y al medio, y lo que es más grave, se está convirtiendo a la nueva dinámica, introducida por las sociedades de conocimiento, en enemiga de la vida y de los humanos.

NECESIDAD DE CREAR NUEVOS PROYECTOS AXIOLÓGICOS COLECTIVOS 
Las religiones, varias veces milenarias, que ejercían como sistemas de valores y de proyecto de vida colectiva, están siendo debilitadas, corroídas y, en algunas regiones del planeta, están prácticamente muertas.

Cuando las religiones mueren o se debilitan, mueren o se debilitan los procedimientos elaborados a lo largo de milenios de cultivo de la cualidad humana y de la cualidad humana profunda y se debilitan o desaparecen las formas de representar y vivir la dimensión absoluta de lo real. Nos quedamos sin maneras acreditadas socialmente de cultivo de esas dimensiones.

Quedamos desmantelados axiológicamente; que cada individuo y cada colectivo salve su cualidad humana, su cualidad humana profunda y su acceso y cultivo a la dimensión absoluta como pueda. El resultado real es que la gran mayoría, siempre en la medida en la que se van introduciendo las nuevas maneras de sobrevivir, no saben y no pueden cultivar convenientemente esas dimensiones del vivir humano.

De forma semejante, los proyectos de vida colectiva, los sistemas axiológicos colectivos, que proporcionaban las ideologías en las sociedades industriales, se están mostrando incapaces de modelar y estructurar los fenómenos que están desencadenando las sociedades de conocimiento. La crisis se está extendiendo.

$\mathrm{Ni}$ las religiones ni las ideologías se muestran capaces de gestionar el nuevo tipo de sobrevivencia que van introduciendo, paso a paso y de forma acelerada, los desarrollos constantes de las ciencias y tecnologías.

Razones para que esto ocurra:

Las sociedades preindustriales eran estáticas, porque vivían, durante milenios, fundamentalmente de la misma forma y porque, para no sufrir riesgos innecesarios, tenían severos procedimientos para bloquear los cambios de importancia.

Las sociedades industriales, aunque cambiaron profundamente, continuaron interpretándose como estáticas, porque aunque reconocían los cambios científicos y técnicos, creían que las formas fundamentales de vida y de organización venían dictadas por la naturaleza misma de las cosas.

Por consiguiente, las sociedades de conocimiento están forzando a transformar radicalmente los sistemas de cohesión y motivación colectiva, los sistemas de programación valoral colectiva.

Exigen que pasemos de sistemas de valores que bloquean el cambio, a sistemas de valores que motiven el cambio. Esta es la mayor transformación que ha sufrido nuestra especie en sus sistemas culturales de sobrevivencia.

Exigen que pasemos de apoyar toda nuestra cultura sobre el individuo, a que la apoyemos sobre los equipos. 
Exigen que pasemos de sociedades jerárquicas y patriarcales, a sociedades no jerárquicas sino de interdependencias y con igualdad completa de hombres y mujeres.

Para esos tránsitos no nos sirven los proyectos axiológicos colectivos de las sociedades preindustriales, que eran las religiones, ni los proyectos axiológicos colectivos de las sociedades industriales, las ideologías.

Las religiones, como instituciones, ya no pueden hacer esa función, porque se fundamentan en creencias que se viven como intocables, por reveladas por Dios. No podemos gestionar sociedades arrastradas por el continuo movimiento en todos los niveles de la vida humana (las sociedades de conocimiento) con programas de vida que exigen bloquear todos los cambios de importancia.

Las ideologías tampoco nos sirven porque comportan interpretar al hombre, a la sociedad, a la economía, y a la política como dictada por la naturaleza de las cosas y, por tanto, no sometidas a cambios profundos.

Las sociedades de conocimiento y sus dinámicas exigen, para poder funcionar coherentemente y con agilidad, la máxima flexibilidad en todos los niveles de la vida individual y colectiva.

Hay que intentar solventar el grave problema que crea esta crisis urgentemente, si no queremos que todo el enorme poder de las dinámicas de las sociedades de conocimiento sea utilizado por los dueños del dinero para su mayor beneficio, en el plazo más corto posible, al precio que sea, para el medio y para el bienestar de los pueblos. Se está manejando las nuevas dinámicas desde la ideología neocapitalista que es propia de las sociedades industriales, pero insistiendo en la investigación, la información (que no es lo mismo que comunicación) y todo ello para una más eficaz explotación.

Nuestros antepasados creyeron que sus proyectos colectivos los recibían de los dioses o de los antepasados sagrados. Luego pensaron que los proyectos axiológicos de vida colectiva venían dictados por la misma naturaleza de las cosas. En ambos casos los proyectos de vida colectiva tenían una procedencia heterónoma.

La dinámica desencadenada por las sociedades de conocimiento nos impide creer en la procedencia heterónoma de nuestros modos de vida. Nos ha hecho ver que los proyectos axiológicos de vida colectiva nos los tenemos que construir nosotros mismos, son, pues, autónomos sin ninguna garantía externa; no tenemos otra garantía que la cualidad humana que hayamos sido capaces de lograr.

En la medida en que las sociedades son afectadas por las dinámicas de las sociedades de conocimiento hay que construir proyectos axiológicos de vida colectiva adecuados. 
Somos de la opinión que no hay que esperar que las dinámicas de las sociedades de conocimiento nos lleguen, porque eso ya indicará que andamos retrasados y con riesgo de ser utilizados por quienes ya están viviendo las sociedades de conocimiento.

Las sociedades de conocimiento evolucionan rápidamente y con ritmo cada vez más acelerado; por esta razón cuanto mayor sea el retraso, mayor será la dificultad para alcanzar el nivel conveniente y no ser marginados. Por la celeridad de los cambios, la marginación puede ser muy peligrosa, porque puede convertirse fácilmente en definitiva.

Para construir nuestros proyectos axiológicos colectivos nos encontramos con un problema: nuestros mayores no nos legaron un saber sobre cómo se construyen esos programas. Ellos, sin duda alguna, los construyeron a lo largo de milenios, pero no fueron conscientes de ello, porque interpretaron, para afianzar su intocabilidad, que los habían recibido. Puesto que los recibieron de los dioses no era ni concebible, ni permitido estudiar cómo estaban construidos. Las sociedades preindustriales los recibieron por revelación de los dioses y de los antepasados sagrados, las sociedades industriales los recibieron igualmente, porque la filosofía creyó desvelar las exigencias de la naturaleza de las cosas.

No nos quedará más solución que estudiar cómo construyeron los proyectos axiológicos colectivos, nuestros antepasados, aunque no lo hicieran conscientemente.

EL DOBLE ACCESO A LA REALIDAD Y SUS CONSECUENCIAS EN LA CREACIÓN DE PROYECTOS AXIOLÓGICOS COLECTIVOS

Para poder construir correctamente nuestros proyectos axiológicos colectivos tendremos que hacer algunas modificaciones en nuestras concepciones.

La primera, y más importante: tendremos que advertir que nuestra estructura lingüística nos proporciona un doble acceso a la realidad: un acceso relativo a nuestras necesidades de vivientes, y otro acceso no relativo a nuestras necesidades, gratuito, absoluto (ab-soluto). Gracias a ese doble acceso somos unos vivientes flexibles respecto al medio.

Los animales, nuestros parientes, tienen un único acceso a lo real. No distinguen entre lo que es significativo para sus vidas y las cosas mismas que tienen ese significado. Identifican las realidades con sus significados. No pueden ser conscientes de que una cosa es la modelación que su programa genético hace de lo real, a la medida de sus necesidades, y otra lo que es modelado, lo que es necesario presupone para hacer posible la modelación. 
Nosotros, gracias a la lengua podemos distinguir entre lo que es el significado que las cosas tienen para nuestras vidas, y las cosas mismas. No identificamos el significado de las realidades con las cosas mismas. Así podemos cambiar el significado de las realidades cuando sea necesario o conveniente.

También tendremos que transformar nuestra antropología. Habrá que pasar de una antropología de cuerpo y espíritu, o de animal y racionalidad, a una antropología que sostiene que somos unos vivientes constituidos como tales por el habla.

La dinámica acelerada de las tecnociencias y sus consecuencias nos exige que nos alejemos de las concepciones antropológicas estáticas (las que defienden que somos un compuesto de cuerpo y espíritu lo es, como también lo es la que sostiene que somos un compuesto de cuerpo y racionalidad), y nos exige que vayamos a una concepción de antropología dinámica: la que sostiene que el hombre es un viviente constituido como tal viviente por el habla. Esa antropología no podrá apoyarse sobre creencias o supuestos filosóficos intocables, sino que deberá esforzarse por apoyarse en datos.

El doble acceso a la realidad, gracias al habla, es un dato; la dimensión relativa de lo real, y su dimensión absoluta también son dato.

Que la dimensión absoluta de lo real sea un dato del vivir humano, transcultural y propio de la peculiar estructura humana de ser vivientes constituidos por el habla, y no fruto de una creencia; que sea un dato sin forma, aunque siempre se aparezca en formas; pero que no esté ligado a unas creencias, unos mitos, unas revelaciones a las que hay que someterse, es de una gran trascendencia cultural, espiritual y ecuménica.

Reconocer, individual y colectivamente, el carácter de dato de la dimensión absoluta, y dato verificable, aunque sea un dato peculiarmente sutil tanto en su noticia como en su verificación, supone una gran transformación de la cultura y de la base de los proyectos axiológicos colectivos de las sociedades.

Plantear la dimensión absoluta como un dato, peculiar en su carácter, pero no como otro piso de la realidad, ni como trascendente ontológico, sino como trascendente a todas nuestras capacidades de conceptualización y representación; no como noticia para un espíritu sino como consecuencia de la estructura misma de un animal constituido por el habla, es de una gran trascendencia cultural y axiológica.

¿Por qué se ha tardado tanto en reconocer su carácter de dato, no ligado a ningún sistema de creencias, ni sumisiones?

Porque en las sociedades preindustriales agrario-autoritarias, de las que inmediatamente procedemos, se necesitaba que la dimensión absoluta, Dios, se apoyara en creencias impositivas que legitimaran la autoridad, la sacralizaran y 
fundamentaran toda la organización jerárquica de la sociedad; organización que era imprescindible para la sobrevivencia de los colectivos.

La categoría fundamental de esas sociedades: "Dios, Señor y Creador y las criaturas" era la categoría básica de la jerarquía, fundamento de la interpretación jerárquica de toda la realidad, del ser y de la organización de ese tipo de sociedades. Para el funcionamiento de esa categoría raíz no resultaba pertinente que Dios fuera único o plural, lo fundamental era la división del orden de lo divino y del orden sometido de las criaturas.

Todo eso está siendo minado por las dinámicas que están desencadenando las sociedades de conocimiento, que necesitan aplanar las organizaciones y apoyarse en la interdependencia de conocimientos y habilidades para la creación de innovaciones científicas, tecnológicas y para la creación de nuevos productos y servicios.

Las nuevas tecnociencias necesitan la creatividad en equipo, y la creación en equipo no casa bien con la sumisión.

Los nuevos proyectos axiológicos colectivos, que vayan respondiendo a los diversos grados de entrada de la dinámica acelerada de las tecnociencias, deberán construirse apoyados en tres factores:

- en las situaciones que crean, en concreto, las tecnociencias y sus consecuencias;

- en el conocimiento de cómo se construyen los proyectos axiológicos colectivos;

- y en una cualidad humana y una cualidad humana profunda adecuada para conducir la marcha de las tecnociencias, y de los productos y servicios que se creen con ellas, al servicio de la vida en general y no en su contra.

Unas sociedades de conocimiento que no arranquen y vayan acompañadas por la cualidad humana serían enormemente dañinas para los colectivos humanos, para el medio, para la continuación de la vida animal y para la misma habitabilidad del planeta.

Hemos de transformar igualmente nuestra epistemología. Tenemos que pasar de una antropología mítica, que pretende que lo que dicen nuestros mitos, teorías y palabras describen la realidad, a una epistemología no mítica que sostiene que nosotros, como los restantes animales, modelamos la realidad a la medida de nuestras necesidades.

El debilitamiento progresivo de la epistemología mítica y su sustitución por una epistemología no mítica, tiene también una enorme trascendencia. La epistemología mítica piensa que las palabras, los mitos y las teorías describen las realidades como son, o se aproximan más y más a como son. La 
epistemología no mítica sabe que nuestras palabras, nuestros mitos y nuestras teorías no describen las realidades como son, sino que las modelan a nuestra medida de vivientes necesitados.

Como los programas genéticos de los animales no pretenden describir la realidad como es, sino modelarla a sus necesidades, así nosotros con nuestras palabras y nuestras culturas no describimos la realidad como es, sino que la modelamos, como los restantes animales. La diferencia es que ellos lo hacen genéticamente y nosotros mediante nuestras construcciones culturales.

La epistemología no mítica imposibilita la fijación de la interpretación y valoración de la realidad; imposibilita los sistemas de creencias que proclaman que la realidad es como ellos afirman; imposibilita los sistemas de valores fijados e intocables; imposibilita la afirmación de que un determinado proyecto axiológico colectivo es el querido y establecido por Dios o por la naturaleza misma de las cosas.

La epistemología no mítica proclama que todo son construcciones propias de un viviente necesitado, en sus diversas formas de sobrevivencia posibilitadas por su doble acceso a la realidad.

Todas nuestras interpretaciones y valoraciones están hechas a la medida de nuestros sensores, nuestra capacidad de acción y a la medida del cerebro de un animal depredador terrestre.

La inmensidad de lo que es, no es a la medida de un pobre viviente necesitado terrestre.

\section{CUALIDAD HUMANA Y CUALIDAD HUMANA PROFUNDA.}

En este contexto, ¿qué entendemos por cualidad humana y qué por cualidad humana profunda?

Entendemos por cualidad humana la conciencia y el cultivo explícito de la doble dimensión de la realidad: la dimensión relativa, y la dimensión absoluta, mediante una enseñanza central de nuestros antepasados:

- el interés por toda la realidad,

- el distanciamiento de nuestros deseos/temores, expectativas y recuerdos,

- y el silenciamiento de todos nuestros patrones de interpretación y valoración. (IDS).

El interés, el distanciamiento y el silenciamiento son tres aspectos de una única actitud.

No hay cualidad humana sin que se de un interés vivo por toda la realidad. Un interés que debe abarcar tanto a nuestra mente, como a nuestro sentir. Al interés desde la mente se le llama simplemente interés; al interés con el sentir se le llama amor.

INTERAÇÕES, BELO HORIZONTE, BRASIL, V.12 N.21, P. 29-44, JAN./JUL. 2017 
Ambos expresan un total volverse a todas las realidades, incluidos nosotros mismos como formando parte de lo real.

Un auténtico interés no puede darse sin que le acompañe la capacidad de distanciamiento de eso mismo por lo que uno se interesa. ¿Distanciamiento de qué? De las propias apetencias con relación a las personas y a los seres; de las propias circunstancias; de los propios deseos, temores, expectativas y recuerdos.

Sólo desde el seno de ese distanciamiento, que es como un paso atrás para ver mejor, que es un desapego de todo lo que no sea el interés por las cosas mismas, puede darse un auténtico dirigirse a las realidades sin otra voluntad que reconocerlas y amarlas.

Ni el interés, ni el desapego son posibles si no van acompañados y precedidos por un silenciamiento de todas nuestros patrones de interpretación y valoración, un distanciamiento de toda referencia a nosotros mismos.

Estos tres aspectos son rasgos de una misma actitud en el que cada uno de los tres elementos necesita de los otros dos para ser posible. El conjunto de los tres es lo que llamamos cualidad humana.

Esa actitud se requiere para todas las operaciones humanas importantes: vida cotidiana bien gestionada, ciencias, artes, filosofía, espiritualidad, etc.

Entendemos por cualidad humana profunda la misma actitud que se requiere para la cualidad humana pero con radicalidad. La cualidad humana está sometida a los condicionamientos del ego, la cualidad humana profunda es incondicional.

Además, en las sociedades de conocimiento hay que someterse a otra tríada:

- la indagación continua con mente y sentir,

- que se deberá realizar en equipo,

- y en servicio de unos a otros, de lo contrario no son posibles los equipos (ICS).

En las sociedades de conocimiento el interés deja de ser un interés sumiso que acepta y se somete a lo que se le concede y se le marca como digno de atención cuidadosa, sino que investiga e indaga libremente.

Sabe que lo que se recibe en interpretación y valoración de la realidad no es como lo real es, sino únicamente una modelación de nosotros mismos, desde las condiciones de sobrevivencia que no son propias.

El interés por toda la realidad le lleva a convertirse en un indagador no sometido, sino libre. Ahora bien, la conciencia de que los conocimientos científicos y tecnológicos de que se dispone son de una enorme complejidad y amplitud le lleva necesariamente a comprender que la indagación que pretende, sólo puede realizarse en equipo. 
Por consiguiente, se dispondrá a indagar en comunicación y colaboración con otros que tengan la misma pretensión. Una comunicación plena, sin reservas, confiada, si no quiere que la investigación quede dañada.

Pero la comunicación plena con otros no es posible si cada uno va a la suya, si cada uno busca, antes que nada, su particular interés. La comunicación sólo se puede conseguir en el seno del servicio de unos a otros; sin ese servicio, también sin reservas, no puede darse la comunicación completa y no puede darse el equipo capaz de llevar adelante la indagación.

En esta tríada, como en la anterior, cada elemento supone a los otros dos para ser posible, y son también tres aspectos de la misma actitud.

\section{LA INDAGACIÓN LIBRE COMO VÍA A LA ESPIRITUALIDAD}

En sociedades que viven de la investigación científica y técnica libre, la aproximación a la dimensión absoluta de lo real y a la cualidad humana y la cualidad humana profunda tiene que ser, inevitablemente, también fruto de una indagación libre. En las sociedades de conocimiento, lo que nuestros antepasados llamaron espiritualidad, no puede realizarse por la vía de la sumisión.

Somos conscientes que la transformación que estamos postulando en el cultivo de la cualidad humana y la cualidad humana profunda (la espiritualidad de nuestros mayores) es el cambio mayor de la historia humana en lo referente a la dimensión absoluta. Es comprensible que existan resistencias de todo tipo muy potentes e intentos de volver atrás y recuperar el pasado.

Oponerse a las sociedades de conocimiento, con la lógica de las transformaciones que provoca, querer frenar su marcha es tarea imposible, ni creo que fuera bueno, supuesto donde hemos llegado, ni para nosotros ni para la vida en el planeta. Hay que seguir adelante y dar una respuesta al cultivo de la cualidad humana y la cualidad humana profunda que sea adecuada a la nueva situación cultural.

¿Cómo se hace una indagación de la dimensión absoluta libre en una sociedad sin creencias, sin un segundo piso de otro mundo, sin religiones y sin dioses?

Hay que estudiar los textos de los sabios para comprender con claridad a qué se refieren cuando hablan de la dimensión absoluta, qué es la cualidad humana y qué la cualidad humana profunda. Ahí no hay nada que creer, ni nada a lo que someterse. Ahí se aprende a indagar correctamente y se comprenden los caminos que pueden conducirnos a desviarnos de la buena indagación.

Sería insensato intentar cultivar la cualidad humana y la cualidad humana profunda inventándolas de nuevo, sin heredar un pasado varias veces milenario. 
La dinámica acelerada e imparable de las ciencias y de las técnicas, en retroalimentación mutua, nos está empujando a una epistemología no mítica que nos hace conscientes de que todas nuestras interpretaciones y valoraciones de la realidad no son descripciones de la realidad más que a partir de nuestros propios modelos y en verdad son modelaciones a nuestra medida. Supuesto eso, no podemos someternos a creencias intocables. Lo que nosotros mismos modelamos, si somos conscientes de ello, no puede someternos.

Las sociedades de conocimiento nos está forzando a excluir todo sistema de creencias - no es que no se quiera creer, es que no se puede-, con ello nos están haciendo un servicio imponderablemente bueno: están fundiendo los dos pisos de la realidad de nuestros antepasados en uno único. Como afirma los pensadores budistas y los sabios hindúes "esto es aquello" y "aquello es esto". Nuestro mundo cotidiano es, a la vez, el mundo de la dimensión absoluta de la realidad.

Las sociedades de conocimiento nos están exigiendo aprender a comprender y vivir todos los fenómenos culturales humanos, en particular esos especialmente sutiles, como son los referentes a la dimensión absoluta y a la cualidad humana profunda, como fenómenos que surgen de nuestra peculiar forma de ser vivientes constituidos como tales por el habla.

No podemos concebir el habla como algo añadido a nuestra condición animal, sino como un invento biológico que permitió que existieran vivientes flexibles frente al medio en el que y del que viven.

Una espiritualidad desde ahí concebida y desde ahí vivida reencanta el mundo y la vida humana. El mundo de las realidades ya no es un mero campo de recursos a explotar y del que vivir, sino que se transforma en sagrado, amable, venerable, sin dualidad. Un mundo de una diversidad inmensa pero uno y sin dualidades.

Recordemos las afirmaciones del cusano cuando afirmaba que el mundo de todas las realidades perecederas no era "otro" de la dimensión absoluta, que la dimensión absoluta no era "otra" del mundo de los seres perecederos: la dimensión absoluta y el mundo de las criaturas son el "no dos".

La dualidad es una construcción de todo viviente porque necesita distinguir y vivir que una cosa es su individualidad, como cuadro de necesidades, y otra el medio en que vive y del que vive. Sin esta modelación dual de la realidad no es posible actuar convenientemente y sobrevivir. Esa es la modelación necesaria de todo viviente, y nosotros estamos sometidos a esa legalidad. Quizás a eso se refiera el mito del pecado original. 
No hay razón ninguna para atribuir esos rasgos de los seres necesitados a la dimensión absoluta de lo real. Los animales, que vienen programados genéticamente en sus formas de vida, tienen que tomar esa modelación como una descripción de lo real, de lo contrario no podrían alimentarse del medio ni procrear. Los humanos, gracias a nuestro doble acceso a la realidad, escapamos de esa fijación y podemos comprender que esa articulación dual de lo real, como sujetos y objetos, es un problema de nuestra necesidad de modelar dualmente, que en realidad la dimensión absoluta es "eso no dual" que también nos incluye a nosotros.

La indagación libre que debe sustituir a la sumisión debe versar no sobre la voluntad de Dios expresada en su revelación, porque la revelación sólo se refiera a lo que es innombrable; ni la indagación debe versar sobre algo que se cree trascendente; ha de hacerse sobre este mundo en el que vivimos: sobre los cielos, la tierra y todo lo que la habita, sobre las aguas y todos los que viven en ellas, sobre nosotros mismos. Cada realidad es un abismo y una gran perplejidad.

Quien quiera investigar la dimensión absoluta debe volverse a la dimensión relativa, porque la dimensión absoluta no se muestra jamás fuera de la dimensión relativa. Indagar las realidades de la dimensión relativa y es indagar la dimensión absoluta. No hay otra posibilidad.

Quien quiera tomar en sus manos la dimensión absoluta, que tome la dimensión relativa.

\section{A MODO DE CONCLUSIÓN}

Estas ideas abren una posibilidad hermosa e inacabable. Estamos en esta vida para ver, sentir, tocar, respetar, amar y venerar este mundo, tal como viene; porque este nuestro frágil mundo, construcción de nuestra torpeza e ignorancia, es la dimensión absoluta al alcance de nuestra indagación, de nuestro ver, de nuestro tocar, respetar, amar y venerar.

Estamos aquí para indagar los cielos, la tierra y todo lo que la habita, las aguas y todo lo que contienen, el misterio de toda existencia y de la inteligencia humana, de su capacidad de sentir, de su capacidad de actuar, incluso de su capacidad de desear, temer, esperar, equivocarse e incluso hacer el mal.

Las flores, los pájaros, los insectos, los animales todos y las plantas son maravillas para nuestra indagación, tan dignas o más que las grandes formaciones estelares. Unos ojos hermosos, la mirada de un niño son como abismos desde donde aflora con fuerza la dimensión absoluta, patente y directamente manifiesta. 
La dimensión relativa, nuestras construcciones y modelaciones, no ocultan la dimensión absoluta, si sabemos indagarla desde el silencio de nosotros mismos, que es el silencio de nuestra proyecciones; cada una de las realidades es, por el contrario, como afirma el Corán, el rostro de Allah, el rostro de la dimensión absoluta que nos mira directamente a los ojos.

¡Qué hermosa es nuestra tarea sobre la tierra! iQué inagotable, apasionante y dulce tarea! Ocuparnos de otra cosa iqué gran necedad!

Dos son las tareas urgentes que nos reclaman las nuevas generaciones:

- aprender a construir los proyectos axiológicos colectivos adecuados siempre a las nuevas circunstancias que se vayan produciendo por la marcha acelerada de las tecnociencias;

- aprender a vivir la gran espiritualidad (la cualidad humana profunda) de nuestros mayores, sin creencias, sin religiones y sin dioses, como una indagación libre y sin fin.

Esas son las tareas que nos están encomendadas, tareas nuevas en la historia de nuestra especie y tareas capitales y muy urgentes.

BIBLIOGRAFÍA

CORBÍ, M. Análisis epistemológico de las configuraciones axiológicas humanas. La necesaria relatividad cultural de los sistemas de valores humanos. Salamanca: Universidad de Salamanca, 1983.

CORBÍ, M. Proyectar la sociedad, reconvertir la religión. Los nuevos ciudadanos. Barcelona: Herder, 1992.

CORBÍ, M. Hacia una espiritualidad laica. Sin creencias, sin religiones, sin dioses. Barcelona: Herder, 2007.

CORBÍ, M. La construcción de los proyectos axiológicos colectivos. Principios de epistemología axiológica 1. Madrid: Bubok, 2013.

CORBÍ, M. La sabiduría de nuestros antepasados para sociedades en tránsito. Principios de epistemología axiológica 2. Madrid: Bubok, 2013.

CORBÍ, M. Protocolos para la construcción de organizaciones creativas de innovación. Principios de epistemología axiológica 3. Madrid: Bubok, 2015.

CORBÍ, M. Cultivo colectivo de la cualidad humana profunda en sociedades de conocimiento globalizada. Principios de epistemología axiológica 4. Madrid: Bubok, 2015.

FERRY, L.; GAUCHET, M. Lo religioso después de la religión. Barcelona: Anthropos, 2006.

GAUCHET, M. El desencantamiento del mundo. Una historia política de la religión. Madrid: Trotta, 2005. 
KRÜGER, K. El concepto de la 'Sociedad del Conocimiento'. Biblio 3W, Revista Bibliográfica de Geografía y Ciencias Sociales, Universidad de Barcelona, Vol. XI, no 683, 25 septiembre 2006. <http://www.ub.es/geocrit/b3w-683.htm>.

NICOLAS de Cues. Du non-outre. Le guide du penseur. Paris: Les éditions du cerf, 2002.

UNESCO Hacia las sociedades del conocimiento Informe Mundial de la Unesco.

Ediciones Unesco, Paris, 2005

http://unesdoc.unesco.org/images/o014/o01419/141908s.pdf 